\title{
Giant single crystal of isotactic polypropylene showing near-equilibrium melting temperature
}

\author{
Harutoshi Asakawa, Koji Nishida, Toshiji Kanaya and Masatoshi Tosaka
}

Giant single crystals of isotactic polypropylene (iPP) were obtained using a specific kinetic pathway through the mesophase of iPP; the pre-quenched iPP mesophase was heated to $166^{\circ} \mathrm{C}$ at a rate of $75^{\circ} \mathrm{C} \mathrm{min}-1$ and maintained at this temperature for $24 \mathrm{~h}$. The obtained crystallites exhibited a very high melting temperature, $T_{\mathrm{m}}$, of $183.7^{\circ} \mathrm{C}$, which is $\sim 19^{\circ} \mathrm{C}$ higher than the iPP's nominal $T_{\mathrm{m}}\left(\sim 165^{\circ} \mathrm{C}\right)$; namely, the observed $T_{\mathrm{m}}$ is immediately close to the literature value of iPP's equilibrium melting temperature, $T_{\mathrm{m}}{ }^{0}\left(186.2{ }^{\circ} \mathrm{C}\right)$. The crystallites have bamboo leaf-like or needle-like morphology and are $\sim 35 \mu \mathrm{m}$ in length and $\sim 3.5 \mu \mathrm{m}$ in width. The thickness of the crystallites was estimated to be $88 \mathrm{~nm}$ according to the melting temperature. The number density of the crystallites in a unit volume was controlled by adjusting the heating rate. As the result, well-isolated single crystals of iPP with the above-mentioned morphologies were obtained.

Polymer Journal (2013) 45, 287-292; doi:10.1038/pj.2012.136; published online 4 July 2012

Keywords: isotactic polypropylene (iPP); mesophase; single crystal

\section{INTRODUCTION}

Single crystals of isotactic polypropylene (iPP), especially large sized crystals, have attracted the interest of researchers for many years. ${ }^{1-12}$ However, the crystal aggregate- the so-called spherulite rather than the single crystal-is usually obtained during the conventional crystallization of iPP from the molten state. Spherulite consists of both crystalline lamella and non-crystalline amorphous regions, which are alternatively arranged with a certain periodicity, that is, long-range order. To obtain thick lamella, considerably long annealing times at high temperatures are usually required; for example, Hikosaka et al. ${ }^{13}$ obtained iPP spherulite with 66 -nm-thick lamellae by annealing at $166^{\circ} \mathrm{C}$ for 6 months. This record value for the lamella thickness resulted in a melting temperature of $\sim 183^{\circ} \mathrm{C},{ }^{14,15}$ which is close to the equilibrium melting temperature, $T_{\mathrm{m}}{ }^{0}$. Several values have been reported for the $T_{\mathrm{m}}{ }^{0}$ of iPP that range from $183.8^{\circ} \mathrm{C}$ to $208^{\circ} \mathrm{C} .{ }^{14-17}$

In addition to the lamella thickness, isolation between the lamellae or the crystallites is another important factor for qualifying the single crystal. 2,410 Although such an isolated single crystal is readily obtained using the solution crystallization method, ${ }^{3-7}$ it is difficult to obtain an isolated single crystal when iPP is crystallized from the bulk melt. Stacks and crosshatches of the lamellae easily occur during the crystallization from the bulk melt. ${ }^{18-22}$ The branching of lamellae also inhibits the formation of a single crysta ${ }^{18}$ and results in the formation of the spherulite. Because of these obstacles, well-isolated iPP single crystals usually only exist during the early stage of the crystallization process. ${ }^{4}, 12$ As a natural consequence, the size of the iPP single crystal is small when iPP is crystallized from the bulk melt.

In contrast to the above-mentioned conventional crystallization of iPP from the melt, we have reported that a rapid heating of iPP from the mesophase yielded large crystallites that have needle-like or 'bamboo leaf-like' (BL) morphology. ${ }^{9} 10$ Because the BL crystal was found by chance, neither the formation mechanism nor the optimization of the formation conditions have been clarified in detail. From a structural point of view, it was initially speculated that the BL crystal is a single crystal and not crystal aggregates, such as spherulite, because it presented very sharp X-ray diffraction peaks compared with the normal melt-crystallized spherulite and showed a monochrome image within each domain of the BL crystal in a polarized microscopy observation. ${ }^{9}$ However, the thermal properties, which directly correlate to the thickness of a single crystal, have not been clarified.

In a previous study, we have observed that a certain temperature region immediately below the melting point with a specific heating rate of $\sim 60-80{ }^{\circ} \mathrm{C} \mathrm{min}^{-1}$ resulted in the maximum growth rate of the crystalline grains when iPP was crystallized from the prequenched mesophase. ${ }^{23}$ Although this result is very useful information for forming BL crystals with good reproducibility, the optimal conditions for the growth rate do not necessarily correspond to the optimal conditions for isolating the single crystal. Therefore, in the present study, by controlling the number density of crystallites in a unit volume, we will clarify the relationship between the melting point of the BL crystal and the formation conditions, such as the heating rate from the mesophase and the annealing temperature.

\section{EXPERIMENTAL PROCEDURE}

Materials and sample preparations

The iPP material was supplied from the Idemitsu Unitech Co., Ltd; the weight-average molecular weight was $M_{\mathrm{w}}=353000$, with a polydispersity of 
$M_{\mathrm{w}} / M_{\mathrm{n}}=4.4$, and a degree of isotacticity (a meso pentad value) of $m m m m=0.98$. This isotacticity is greater than the critical value $(0.68)$ of isotacticity required to form the mesophase. ${ }^{24}$ Sample films with a thickness of $200 \mu \mathrm{m}$ were prepared by quenching molten iPP to $0{ }^{\circ} \mathrm{C}$ by dipping the films into ice water. The prepared films were macroscopically transparent and presented two broad peaks in a wide-angle X-ray diffraction (WAXD) pattern, which are characteristic of mesophase iPP. ${ }^{25-27}$

\section{Time-temperature protocol}

As mentioned in the introduction, the formation conditions for the BL crystal were narrowed in the previous study; specifically, a certain temperature region immediately below the melting point and a specific heating rate of $\sim 60-80{ }^{\circ} \mathrm{C} \mathrm{min}^{-1}$ resulted in the maximum growth rate of the crystalline grain. ${ }^{23}$ In this study, we prepared two groups of time-temperature protocols based on the above-mentioned condition: first, the heating rate was fixed at $75^{\circ} \mathrm{C} \mathrm{min}^{-1}$ and the final annealing temperature was scanned (Figure 1a); and second, the final annealing temperature was fixed at $164^{\circ} \mathrm{C}$ and the heating rate was scanned (Figure 1b). In both protocols, the final annealing period was fixed to $24 \mathrm{~h}$.

\section{Optical microscopy observation}

Observations with an optical microscope were performed using an Optiphot2Pol, a product of Nikon, Tokyo, Japan. The bright-field mode was used to avoid counting loss in the number of crystallites because only the crystallites arranged in a specific orientation are detected in the polarized mode. The prequenched sample films were heated and annealed according to the prescribed protocol (Figure 1) using a laboratory-made hot stage. The hot stage consisted of a heater block and a control unit. The heater block, to which the sample was in contact, was controlled by a programmable CPU unit, KV-3000 and a PID unit, KV-TF40; both the CPU and the PID units were products of KEYENCE, Osaka, Japan. The temperatures presented in the results were the calibrated temperatures using the actual measured temperatures at the sample position.

\section{Differential scanning calorimetry}

Differential scanning calorimetry (DSC) measurements were performed using a Diamond DSC, a product of Perkin-Elmer, Waltham, MA, USA. The prequenched sample films were heated and annealed within the DSC instrument according to the prescribed protocol (Figure 1), similar to the optical microscope observations. Immediately after the annealing, the DSC data were recorded during the heating process at a rate of $10^{\circ} \mathrm{Cmin}^{-1}$. In the latter
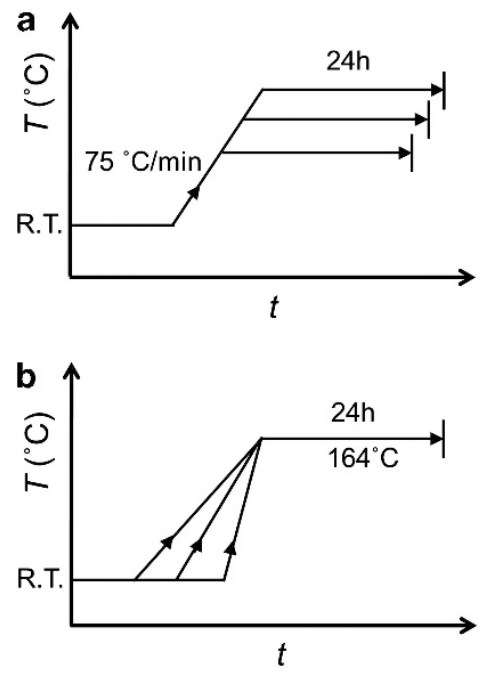

Figure 1 Time-temperature protocols. The heating rate was fixed at $75^{\circ} \mathrm{C} \mathrm{min}^{-1}$ and the final annealing temperature was scanned (a), and the final annealing temperature was fixed at $164^{\circ} \mathrm{C}$ and the heating rate was scanned (b). heating process, the melting behavior of the crystallites was observed. The temperature was calibrated from the melting temperatures of pure In and Sn. All of the measurements were performed under a nitrogen atmosphere.

\section{Wide-angle X-ray diffraction}

WAXD measurements were performed using a NANO-Viewer, a product of Rigaku, Tokyo, Japan, with an imaging plate detector at a sample-to-detector distance of $44.5 \mathrm{~mm}$.

\section{RESULTS AND DISCUSSION}

\section{Effect of annealing temperature on crystallization}

Figure 2 presents selected optical microscope images after the samples were annealed for $24 \mathrm{~h}$ at various temperatures $\left(152-172{ }^{\circ} \mathrm{C}\right)$ following heating at a rate of $75^{\circ} \mathrm{C} \mathrm{min}-1$, according to the prescribed protocol (Figure 1a).

As observed in Figure 2, the crystalline entities were only detected when the samples were annealed within a specific temperature window of $157-164^{\circ} \mathrm{C}$. Within the specific temperature window,

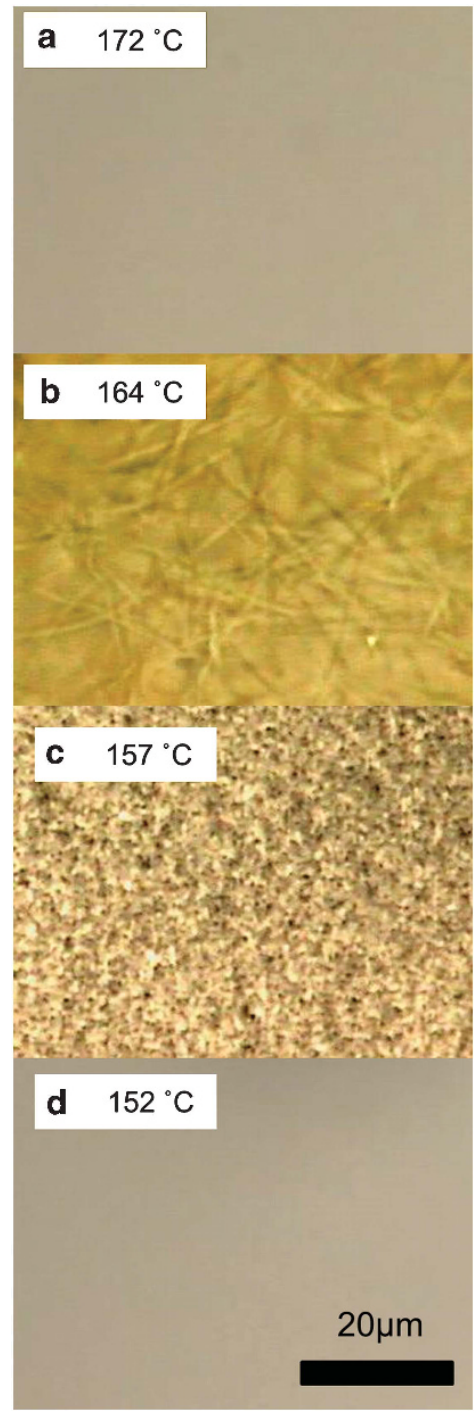

Figure 2 Selected optical microscopy images after the samples were annealed for $24 \mathrm{~h}$ at various temperatures (172 (a), 164 (b), 157 (c) and $152^{\circ} \mathrm{C}$ (d)) following heating at a rate of $75^{\circ} \mathrm{C} \mathrm{min}^{-1}$. 
the size and number density of the crystallites were considerably dependent on the annealing temperature; well-isolated large $\mathrm{BL}$ crystals were observed when the sample was annealed at $164{ }^{\circ} \mathrm{C}$ (Figure 2b), whereas densely populated small crystallites, although not clear because of overlapping, were observed when the sample was annealed at $157^{\circ} \mathrm{C}$ (Figure 2c). On the outer boundaries of the specific temperature window, the images were transparent. The reasons for the transparency below and above the specific window are considered to be different, as follows. Above the specific window $\left(172{ }^{\circ} \mathrm{C}\right.$, Figure $\left.2 \mathrm{a}\right)$, the transparency is unquestionably due to the complete melting, that is, non-existence of crystallites, whereas below the specific window $\left(152^{\circ} \mathrm{C}\right.$, Figure $\left.2 \mathrm{~d}\right)$, the absence of the detectable entities does not mean the non-existence of crystallites. At $152^{\circ} \mathrm{C}$, the majority of the mesophase actually transforms into the $\alpha$-crystal; ${ }^{10,28,29}$ however, the $\alpha$-crystal exists in the nodular form with a diameter of several tens of nm at most, ${ }^{29}$ that is, the dimension of the structure is far less than the optical wavelength. Consequently, the iPP film consisting of such nano-crystallites is macroscopically transparent. ${ }^{30}$ On the basis of Figure 2, well-isolated large BL crystals are obtained when the pre-quenched iPP mesophase is annealed at $\sim 164{ }^{\circ} \mathrm{C}$ following heating at a rate of $75^{\circ} \mathrm{C} \mathrm{min}{ }^{-1}$.

\section{Effect of heating rate on crystallization}

Figure 3 presents selected optical microscope images after the samples were annealed for $24 \mathrm{~h}$ at $164{ }^{\circ} \mathrm{C}$ following heating at various rates (5-108 $\left.{ }^{\circ} \mathrm{C} \mathrm{min}^{-1}\right)$, according to the prescribed protocol (Figure $1 \mathrm{~b}$ ). As observed in Figure 3, the crystalline entities were detected when the heating rate was greater than $20^{\circ} \mathrm{C} \mathrm{min}^{-1}$. At a heating rate of $5^{\circ} \mathrm{C} \min ^{-1}$, the images were transparent (Figure $3 \mathrm{a}$ ). The reason for the transparency is not the non-existence of crystallites but the formation of the nano-crystallites, similar to the case in Figure $2 \mathrm{~d}$. Densely populated small crystallites, although not clear because of overlapping, were observed when the sample was heated at a rate of $20{ }^{\circ} \mathrm{C} \mathrm{min}^{-1}$ (Figure $3 \mathrm{~b}$ ). At a heating rate of $39^{\circ} \mathrm{C} \mathrm{min}^{-1}$, fairly wellisolated large $\mathrm{BL}$ crystals were observed (Figure 3c). With increasing heating rate, the isolation between neighboring crystallites was improved $\left(75^{\circ} \mathrm{C} \mathrm{min}^{-1}\right.$, Figure $\left.2 \mathrm{~b}\right)$. Finally, a unique isolated $\mathrm{BL}$ crystal within a scope was attained when the heating rate reached $108^{\circ} \mathrm{C} \mathrm{min}^{-1}$ (Figure 3d). At present, we have not yet succeeded in extracting the crystallite from the matrix; the matrix in this case is the mesophase when the crystallization process is stopped by quenching. In this sense, we should say, 'a single crystal embedded in the mesophase.'

When the annealing temperature was fixed at $164^{\circ} \mathrm{C}$, the heating rate was demonstrated to be an effective parameter for controlling the isolation and the morphology of the crystallites for the crystallization of iPP from the mesophase. Furthermore, note that the annealing temperature of $164{ }^{\circ} \mathrm{C}$ is considered to be considerably high in the conventional sense because at such a high temperature it takes a very long time for iPP to crystallize from the normal molten state. ${ }^{31}$ One of the characteristics of crystallization from the mesophase is that the crystallization proceeds properly at such a high temperature. Another mechanism for crystallization from the mesophase is discussed below.

In what follows, the size and number density of the obtained crystallites are discussed quantitatively on the basis of the image analysis of the micrographs. First, we determined the average longitudinal and lateral sizes, which were apparently determined from the micrographs. Because the crystallites were far thinner than the resolution of the optical microscope, we discuss it below on the basis of the DSC analysis. Figure 4 shows a representation of the sizedistribution histogram when iPP was crystallized at $164{ }^{\circ} \mathrm{C}$ following

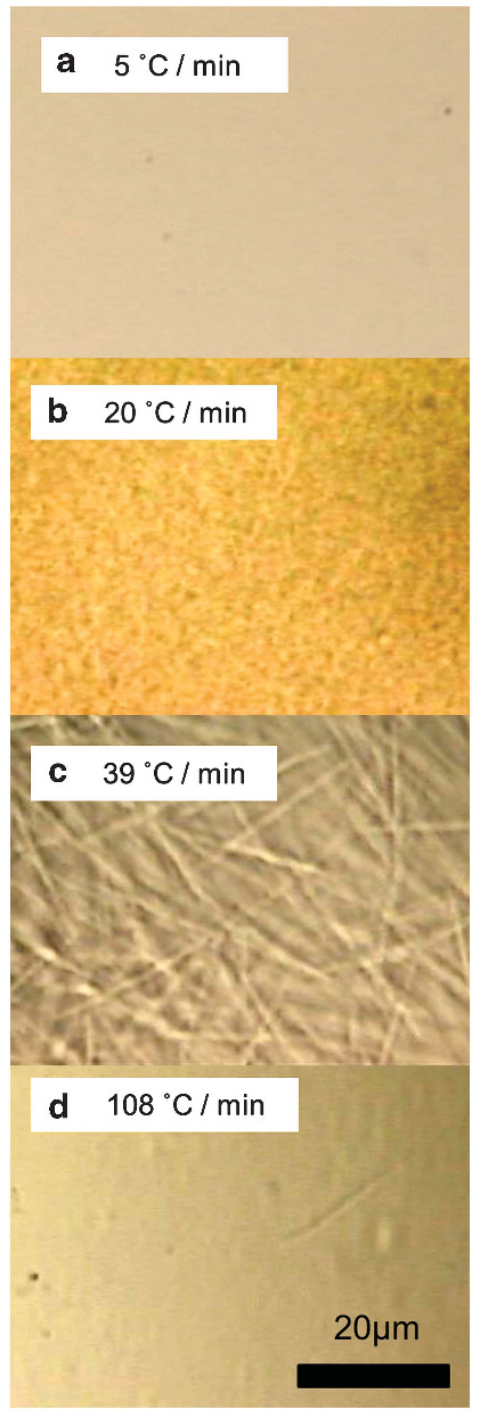

Figure 3 Selected optical microscopy images after the samples were annealed for $24 \mathrm{~h}$ at $164^{\circ} \mathrm{C}$ following heating at various rates (5 (a), 20 (b), 39 (c) and $108{ }^{\circ} \mathrm{Cmin}^{-1}$ (d)).

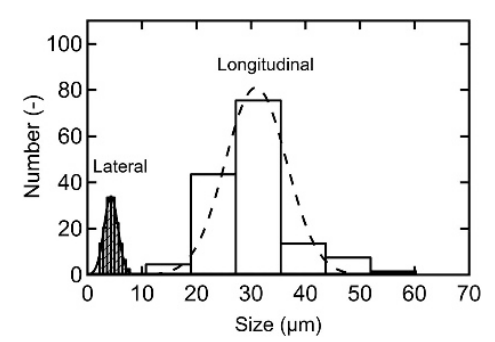

Figure 4 Size-distribution histogram of crystallites when iPP was crystallized at $164{ }^{\circ} \mathrm{C}$ following heating from the mesophase at a rate of $75^{\circ} \mathrm{C} \min ^{-1}$.

heating from the mesophase at a rate of $75^{\circ} \mathrm{Cmin}^{-1}$. The condition corresponds to Figure $2 \mathrm{~b}$, but the sampling was performed covering a wider scope than the displayed microscope image to obtain reasonable statistics. By fitting the Gaussian function to the histogram, the average and standard deviation of the longitudinal and lateral sizes were obtained. 
The longitudinal and lateral sizes of the BL crystals as a function of the heating rate are shown in Figures $5 \mathrm{a}$ and b, respectively. With increasing heating rate, the longitudinal size increased from $\sim 7$ to $\sim 35 \mu \mathrm{m}$. In parallel, the lateral size slightly increased with increasing heating rate, although the quantitative value for the thinnest case (heating rate of $20^{\circ} \mathrm{C} \mathrm{min}^{-1}$ ) could not be determined because of the resolution of the microscope.

The aspect ratio of the BL crystals as a function of the heating rate was estimated by dividing the longitudinal size by the lateral size, and this ratio is shown in Figure 6. Although the aspect ratio increased with the increasing heating rate, it showed inherently high values of $\sim 5-10$, in common with the case of the single crystals that were produced during the early stage of the melt-crystallization. ${ }^{12}$

The number density in a unit area was estimated by simply counting the number of crystallites within a unit area in the micrographs. Assuming that only the crystalline entities distributed within the focal depth were detected, the number density in a unit volume was calculated by dividing the number density in a unit area by the focal depth; the focal depth of the present optics was estimated to be $6.2 \mu \mathrm{m}$ based on the numerical aperture of the lens. Figure 7 shows the estimated number density of the crystallites in a unit volume. The number density varied more than two orders of magnitude with increasing heating rate until $\sim 60{ }^{\circ} \mathrm{C} \mathrm{min}^{-1}$. Therefore, the heating rate was shown to be an effective parameter for controlling the number density of the crystallites; specifically, the isolation between the single crystals can be controlled by the heating rate in the present specific crystallization of iPP from the mesophase. A further increase in the heating rate slightly decreased the number density.

\section{Effect of annealing temperature on the melting behavior of the obtained BL crystals}

Figure 8 presents selected thermograms from the DSC measurements during heating scans at a rate of $10{ }^{\circ} \mathrm{Cmin}^{-1}$ for the samples annealed within the DSC instrument according to the prescribed protocol (Figure 1a) at various temperatures $\left(130-167^{\circ} \mathrm{C}\right)$ for $24 \mathrm{~h}$. The heating scans were started from the prescribed annealing temperatures to $200{ }^{\circ} \mathrm{C}$ immediately after the annealing processes. As observed in Figure 8, the endothermic melting peak shifted toward higher temperatures with increasing annealing temperature.

In Figure 9, the melting temperature $T_{\mathrm{m}}$ is plotted as a function of the annealing temperature or the crystallization temperature $T_{\mathrm{c}}$, the so-called Hoffman-Weeks plots. The diagonal thick solid line indicates the relation of $T_{\mathrm{m}}=T_{\mathrm{c}}$. In normal Hoffman-Weeks plots,

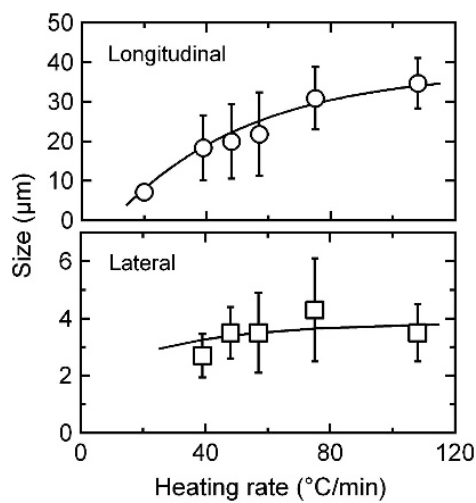

Figure 5 Longitudinal (a) and the lateral (b) sizes of the BL crystals as a function of the heating rate.

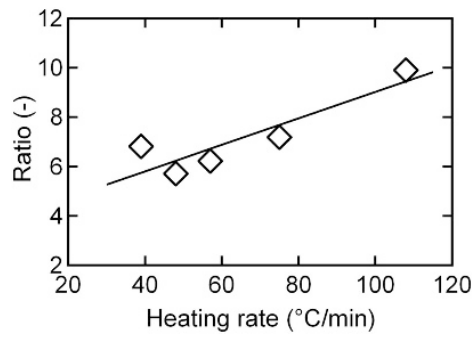

Figure 6 Aspect ratio of the BL crystals as a function of the heating rate.

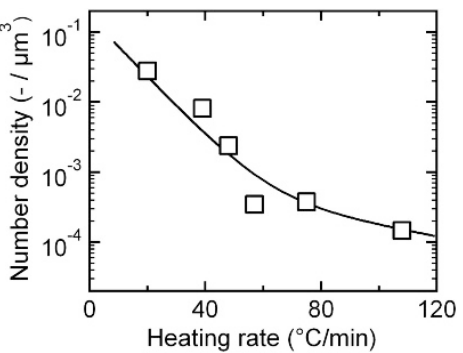

Figure 7 Number density of the crystallites in a unit volume as a function of the heating rate.

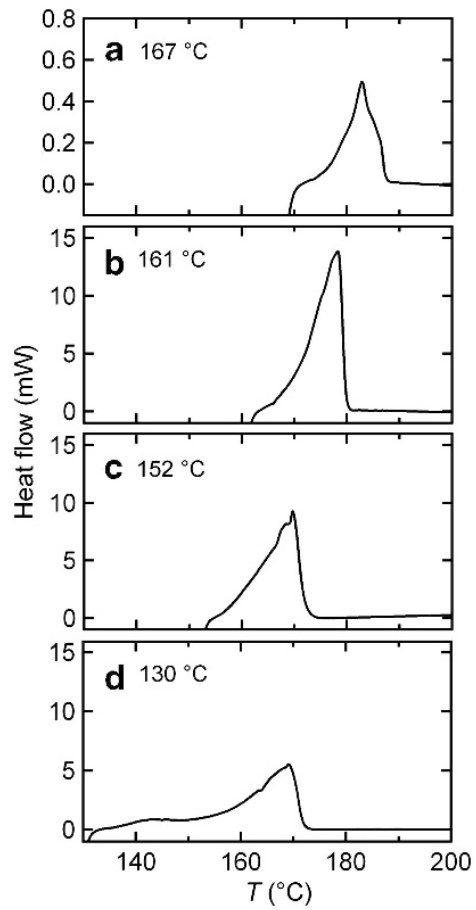

Figure 8 Selected DSC thermograms during heating scans at a rate of $10^{\circ} \mathrm{C} \mathrm{min}^{-1}$ for the samples annealed within the DSC instrument at various temperatures (167 (a), 161 (b), 152 (c) and $\left.130{ }^{\circ} \mathrm{C}(\mathbf{d})\right)$.

$T_{\mathrm{m}}$ is often linearly proportional to $T_{\mathrm{c}}$ or near-linearly in the region above the $T_{\mathrm{m}}=T_{\mathrm{c}}$ line, and the intersection with the $T_{\mathrm{m}}=T_{\mathrm{c}}$ line is considered to give the $T_{\mathrm{m}}{ }^{0.32}$

It is clear in Figure 9 that the present behavior is quite different from the normal behavior of the above-mentioned Hoffman-Weeks plots. Paradoxically speaking, the fact that one cannot draw single 
linear line in the Hoffman-Weeks plots indicates the existence of different crystal forms, and accordingly, the existence of different crystallization mechanisms. In this sense, we should say that Figure 9 is the 'apparent' Hoffman-Weeks plot. Below $T_{\mathrm{c}}=\mathrm{ca} .155^{\circ} \mathrm{C}, T_{\mathrm{m}}$ was almost independent of $T_{\mathrm{c}}$. As previously discussed, the transparency of the micrograph obtained at $152{ }^{\circ} \mathrm{C}$ (Figure 2d) indicates that the crystallites in this temperature region exist in the form of a nodule. Therefore, the $T_{\mathrm{m}}$ is restricted by the nodular size. Above $T_{\mathrm{c}}=\mathrm{ca}$. $155^{\circ} \mathrm{C}, T_{\mathrm{m}}$ increased rapidly and seems to plateau at $\sim 185^{\circ} \mathrm{C}$. The gradient of the $T_{\mathrm{m}}$ vs $T_{\mathrm{c}}$ plots is an index of the perfection and morphology of the crystal; ${ }^{32-35}$ with the crystal approaching the infinitely thick or the limiting thickness due to the chain structure, the gradient decreases and becomes horizontal in the ultimate case. Therefore, the observed plateau behavior is the indication that the present $\mathrm{BL}$ crystal is near the certain limitation, hopefully an infinitely thick crystal.

The relationships between the $T_{\mathrm{m}}$ and the thickness of the crystallites have been discussed quantitatively in literature. ${ }^{14-16}$ Here, we estimated the thickness of the $\mathrm{BL}$ crystals assuming the following relationship proposed by Yamada et al. ${ }^{14,15}$

$$
T_{\mathrm{m}}=186.2-220 /\langle l\rangle
$$

The values of $T_{\mathrm{m}}$ are dependent on the methods, such as optical microscopy and DSC. In this study, we determined the $T_{\mathrm{m}}$ using DSC; therefore, the thickness of the crystallites $\langle l\rangle$ is an averaged thickness weighted by the DSC. Figure 10 shows an estimated value of $\langle l\rangle$ as a function of the annealing temperature, $T_{\mathrm{c}}$. The $\langle l\rangle$ was almost independent of $T_{\mathrm{c}}$ below $T_{\mathrm{c}}=\mathrm{ca} .155^{\circ} \mathrm{C}$ and the behavior of $T_{\mathrm{m}}$, whereas the $\langle l\rangle$ showed a tendency to diverge above $T_{\mathrm{c}}=\mathrm{ca} .155^{\circ} \mathrm{C}$ in contrast to the behavior of $T_{\mathrm{m}}$. Apart from the diverging tendency, we have attained a crystal thickness of $88 \mathrm{~nm}$. Although the obtained

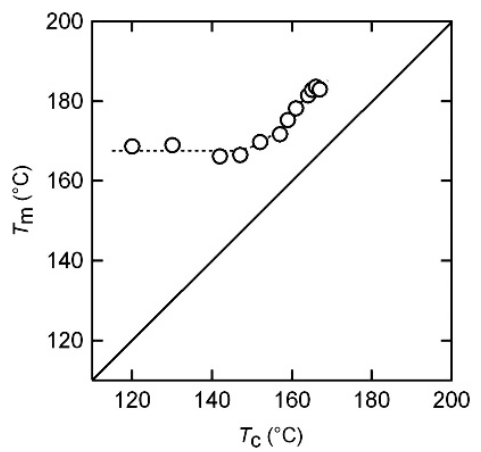

Figure 9 Melting temperature, $T_{m}$, as a function of the annealing temperature, $T_{\mathrm{c}}$.

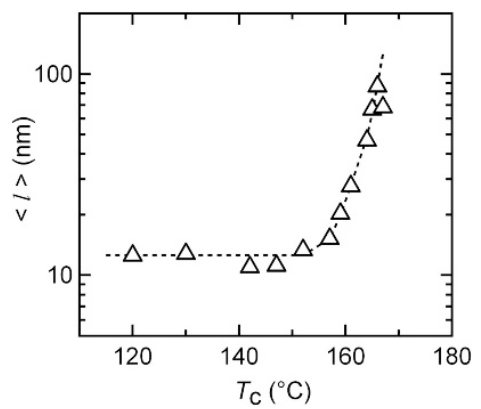

Figure 10 Thickness of the crystallites, $\langle I\rangle$, as a function of the annealing temperature, $T_{\mathrm{c}}$. value is similar to or slightly larger than the reported value of the lamella thickness of $66 \mathrm{~nm}$ when crystallized from the melt, ${ }^{15}$ the present annealing process greatly reduced the annealing time; the annealing time required $24 \mathrm{~h}$ when crystallized from the mesophase, whereas it required 6 months when crystallized from the melt.

\section{Crystal form of giant single crystal}

Figure 11 shows the WAXD pattern from the sample obtained by annealing at 164 and $135^{\circ} \mathrm{C}$. The peak at $\sim 2 \theta=31.6^{\circ}$ arises from the diffraction from the $\alpha-2$ form. ${ }^{17}$ It is obvious from this figure that the crystal form for the lower temperature (nodular crystals) is $\alpha-1$ and that of the higher temperature (BL crystals) is the $\alpha-2$. The unusual behavior in the Hoffman-Weeks plots in Figure 9 should be associated with the transition from the $\alpha-1$ to the $\alpha-2$ form.

\section{Possible mechanism for the formation of a giant single crystal}

The formation conditions and characteristics of the BL crystals have been substantially elucidated as thus far described. Finally, the possible mechanism for forming the BL crystals is discussed.

In the $\alpha$-crystal of iPP, the branching, which results in the formation of spherulite, is induced by a rupture of the sequence of alternating left- and right-handed helices. ${ }^{36}$ In general, annealing at higher temperatures is more favorable for producing defect-free crystals. ${ }^{37}$ Accordingly, (i) the selection process of the helix-sense and (ii) annealing at a temperature as high as possible must be essential for obtaining a giant single crystal of iPP.

The requisite (i) will be satisfied by the multiple steps of the crystallization from the mesophase. Because left- and right-handed helices are randomly distributed in the mesophase of $i \mathrm{PP},{ }^{38}$ translational rearrangements of both-handed helices are necessary to form the defect-free $\alpha$-crystal. In the course to obtain the BL crystals from the mesophase, iPP experiences many steps that are as follows: first, the mesophase transforms into the crystalline phase within the nodule. Consecutively, the size of the nodule increases by repeating the partial melting and recrystallization. There is a distribution in nodule size, ${ }^{39}$ and there is also likely a distribution in the rate of defects. Such distributions facilitate the partial melting and recrystallization because the larger and the less defective nodular crystals survive and grow by consuming the smaller and the more defective nodular crystals in the heating and the annealing processes. Such multiple steps, including the partial melting and recrystallization, will provide enough opportunities to select the helix-sense. There should be an optimal heating rate that is favorable for such recrystallization. ${ }^{23}$

The crystallization from the mesophase also facilitates requisite (ii). It is a matter of course that the nucleation at very high temperatures

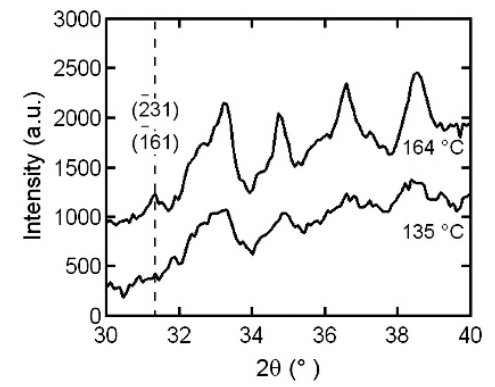

Figure 11 WAXD pattern from the sample obtained by annealing at 164 and $135^{\circ} \mathrm{C}$. 
is a difficult process without the appropriate nucleus. Fortunately, the crystallization from the mesophase provides the nucleus, which has the advantage of having fewer defects, as discussed in the previous paragraph. Such a nucleus with few defects strongly assists the crystallization process in the high temperature region.

These advantages of the crystallization from mesophase will enable the formation of a large single crystal with fewer defects.

\section{CONCLUSION}

Giant single crystals of iPP have been obtained using a specific kinetic pathway through the mesophase. The obtained crystals possess a large size without branching, a high aspect ratio, isolation and a high melting temperature. These characteristics demonstrate the quality of the single crystal. In addition to the quality, the present crystals are obtained using a relatively short annealing period.

1 Ranby, B. G., Morhead, F. D. \& Malter, N. M. Morphology of n-alkanes, linea polyethylene, and isotactic polypropylene crystallized from solution. J. Polym. Sci. 44, 349-367 (1960)

2 Geil, P. H. Folded molecules in lamellas crystallized from molten polymers. J. Appl. Phys. 33, 642-643 (1962).

3 Sauer, J. A., Morrow, D. R. \& Richardson, G. C. Morphology of solution-grown polypropylene crystal aggregates. J. Appl. Phys. 36, 3017-3021 (1965)

4 Khoury, F. The spherulitic crystallization of isotactic polypropylene from solution: On the evolution of monoclinic spherulites from dendritic chain-folded crystal precursors. J. Res. Natl. Bur. Stand. Sect. A 70, 29-61 (1966).

5 Kojima, M. Morphology of polypropylene crystal. I. Dilute solution-grown lamellar crystals. J. Polym. Sci., Part A-2: Polym. Phys. 5, 597-613 (1966).

6 Kojima, M. Morphology of polypropylene crystal. II. Twinning of lamellar crystals. J. Polym. Sci., Part A-2: Polym. Phys. 5, 615-622 (1966).

7 Padden, F. J. \& Keith, H. D. Crystallization in thin films of isotactic polypropylene. J. Appl. Phys. 37, 4013-4020 (1966).

8 Padden, F. J. \& Keith, H. D. Mechanism for lamellar branching isotactic polypropylene. J. Appl. Phys. 44, 1217-1223 (1973).

9 Nishida, K., Konishi, T., Kanaya, T. \& Kaji, K. Novel morphology of isotactic polypropylene crystal generated by a rapid temperature jump method. Polymer 45, 1433-1437 (2004).

10 Konishi, T., Nishida, K. \& Kanaya, T. Crystallization of isotactic polypropylene from prequenched mesomorphic phase. Macromolecules 39, 8035-8040 (2006).

11 Zhao, J., Qiu, J., Niu, Y. \& Wang, Z. Evolutions of morphology and crystalline ordering upon annealing of quenched isotactic polypropylene. J. Polym. Sci., Part B: Polym. Phys. 47, 1703-1712 (2009)

12 Yamada, K., Kajioka, H., Nozaki, K. \& Toda, A. Morphology and growth of single crystals of isotactic polypropylene from the melt. J. Macromol. Sci., Part B: Phys. 50, 236-247 (2010)

13 Maiti, P., Hikosaka, M., Yamada, K., Toda, A. \& Gu, F. Lamellar thickening in isotactic polypropylene with high tacticity crystallized at high temperature. Macromolecules 33, 9069-9075 (2000)

14 Yamada, K., Hikosaka, M., Toda, A., Yamazaki, S. \& Tagashira, K. Equilibrium melting temperature of isotactic polypropylene with high tacticity: 1 . Determination by differential scanning calorimetry. Macromolecules 36, 4790-4801 (2003).

15 Yamada, K., Hikosaka, M., Toda, A., Yamazaki, S. \& Tagashira, K. Equilibrium melting temperature of isotactic polypropylene with high tacticity: 2 . Determination by optical microscopy. Macromolecules 36, 4802-4812 (2003).
16 Cheng, S. Z. D., Janimak, J. J., Zhang, A. \& Hsieh, E. T. Isotacticity effect on crystallization and melting in polypropylene fractions: 1 . Crystalline structures and thermodynamic property changes. Polymer 32, 648-655 (1991).

17 Nakamura, K., Shimizu, S., Umemoto, S., Thierry, A., Lotz, B. \& Okui, N. Temperature dependence of crystal growth rate for $a$ and $b$ forms of isotactic polypropylene. Polym. J. 40, 915-922 (2008).

18 Norton, D. R. \& Keller, A. The spherulitic and lamellar morphology of melt-crystallized isotactic polypropylene. Polymer 26, 704-716 (1985).

19 Vaughan, A. C. \& Basset, D. C. Early stages of spherulite growth in melt-crystallized polystyrene. Polymer 29, 1397-1401 (1988).

20 Olley, R. H. \& Bassett, D. C. On the development of polypropylene spherulites. Polymer 30, 399-409 (1989).

21 Janimak, J. J., Cheng, S. Z. D., Giusti, P. A. \& Hsieh, E. T. Isotacticity effect on crystallization and melting in polypropylene fractions: 2 . Linear crystal growth rate and morphology study. Macromolecules 24, 2253-2260 (1991).

22 Yamada, K., Matsumoto, S., Tagashira, K. \& Hikosaka, M. Isotacticity dependence of spherulitic morphology of isotactic polypropylene. Polymer 39, 5327-5333 (1998).

23 Asakawa, H., Nishida, K., Yamamoto, J., Inoue, R. \& Kanaya, T. Heating rate effects on the crystallization behavior of isotactic polypropylene from mesomorphic phase-a de-polarized light transmission study. Polymer 53, 2777-2782 (2012).

24 Konishi, T., Nishida, K., Kanaya, T. \& Kaji, K. Effect of isotacticity on formation of mesomorphic phase of isotactic polypropylene. Macromolecules 38, 8749-8754 (2005).

25 Natta, G. Progress in five years of research in stereospecific polymerization. SPE. J. 15 373-382 (1959).

26 Miller, R. L. On the existence of near-range order in isotactic polypropylenes. Polymer 1, 135-143 (1960)

27 Nishida, K., Okada, K., Asakawa, H., Matsuba, G., Ito, K., Kanaya, T. \& Kaji, K. In situ observations of the mesophase formation of isotactic polypropylene-a fast time-resolved X-ray diffraction study. Polym. J. 44, 95-101 (2012).

28 Zia, Q. Androsch, R., Radusch, H.- J. \& Piccarolo, S. Morphology, reorganization and stability of mesomorphic nanocrystals in isotactic polypropylene. Polymer 47, 8163-8172 (2006)

29 Zia, Q., Radusch, H.- J. \& Androsch, R. Direct analysis of annealing of nodular crystals in isotactic polypropylene by atomic force microscopy, and its correlation with calorimetric data. Polymer 48, 3504-3511 (2007).

30 Funaki, A., Kanai, T., Saito, Y. \& Yamada, T. Analysis of Contributing factors to production of highly transparent isotactic polypropylene extrusion sheets. Part I. Polym. Eng. Sci. 50, 2356-2365 (2010).

31 Radhakrishnan, J., Ichikawa, K., Yamada, K., Toda, A. \& Hikosaka, M. Nearly pure $\alpha 2$ form crystals obtained by melt crystallization of high tacticity isotactic polypropylene. Polymer 39, 2995-2997 (1998).

32 Hoffman, J. D. \& Weeks, J. J. Melting process and the equilibrium melting temperature of polychlorotrifluoroethylene. J. Res. Natl. Bur. Stand., Sect. A 66, 13-27 (1962).

33 Baker, C. H. \& Mandelkern, L. The crystallization and melting of copolymers I-The effect of the crystallization temperature upon the apparent melting temperature of polymethylene copolymers. Polymer 7, 7-21 (1966).

34 Okui, N. \& Kawai, T. Crystallization of ethylene/vinylacetate random copolymers. Makromol. Chem. 154, 161-176 (1972).

35 Yoo, H. Y., Umemoto, S., Kikutani, T. \& Okui, N. Co-crystallization behaviour and melting point depression in poly(ethylene terephtalate-co-1,4-cyclohexylene dimethylene terephthalate) random copolyesters. Polymer 35, 117-122 (1994).

36 Lotz, B. \& Wittmann, J. C. The molecular origin of lamellar branching in the (monoclinic) form of isotactic polypropylene. J. Polym. Sci., Part B: Polym. Phys. 24 1541-1558 (1986)

37 Bassett, D. C. \& Olley, R. H. On the lamellar morphology of isotactic polypropylene spherulites. Polymer 25, 935-934 (1984).

38 Corradini, P., De Rosa, C., Guerra, G. \& Pettracone, V. Comments on the possibility that the mesomorphic phase of isotactic polypropylene is composed of small crystals of the b crystalline form. Polym. Commun. 30, 281-285 (1989).

39 Ogawa, T., Miyaji, H. \& Asai, K. Nodular structure of polypropylene. J. Phys. Soc., Jpn. 54, 3668-3670 (1985). 